\section{Recent advances in cortical visual impairment}

\author{
William V Good* MD, Smith-Kettlewell Eye Research \\ Institute, San Francisco, California, USA; \\ James E Jan MD, Vancouver Children's Hospital, British \\ Columbia, Canada; \\ Susan K Burden BS, University of Michigan Medical School, \\ Ann Arbor, Michigan; \\ Ann Skoczenski PhD, University of Massachusetts Medical \\ School, Massachusetts; \\ Rowan Candy PhD, Smith-Kettlewell Eye Research Institute, \\ San Francisco, California, USA.
}

*Correspondence to first author at Smith-Kettlewell Eye Research Institute, 2318 Fillmore Street, San Francisco, CA 94115, USA.

E-mail:good@skivs.ski.org

Cortical visual impairment (CVI) is the leading cause of bilateral visual impairment in children in Western countries ${ }^{1-3}$. This finding reflects better methods for identifying visual impairment due to CNS injury and also advances in perinatal care, which have increased the survival rate of children with neurological morbidity. This review will describe advances in the diagnosis and management of CVI. Central to our discussion is a definition of CVI that includes a decrease in visual acuity. New treatment and rehabilitative measures are badly needed for this disorder. We hope to stimulate interest in CVI, a disease that has become a significant public health problem.

The incidence of CVI is increasing ${ }^{3}$. In a study of five Nordic countries, Rosenberg and coworkers ${ }^{5}$ noted that brain damage accounts for a growing number of cases of childhood visual impairment. They suggested that better medical care has lowered the mortality rate of children with severe medical problems. Although CVI alone is not life threatening, its associated neurological disorders may have been fatal in the past. In one study from Chile, $2.1 \%$ of children enrolled in schools for the blind, who are either visually impaired or blind, had visual diagnoses involving CVI, although this may be an underestimate $^{6}$. In another study from Liverpool, Rogers ${ }^{7}$ found that CVI was the most common cause of visual impairment in children with associated neurological disorders (49\% of the study population). The Oxford Register of Early Childhood Impairments ${ }^{8}$ reports the overall incidence of bilateral vision impairment at $0.14 \%$, with $29.5 \%$ of cases due to CVI and $14.1 \%$ due to nystagmus: the second major cause of impairment in this study population. In Northern California, CVI was also found to be the leading cause of visual impairment in children under the age of 5 years?

Visual deficits in cortical visual impairment

CVI is a neurological disorder resulting in bilateral impairment of visual acuity caused by damage to the CNS, meaning visual acuity is reduced as a result of non-ocular disease ${ }^{3}$. Visual acuity is a measure of the ability to visually resolve objects of interest in detail. One of the remarkable aspects of CVI is the near universal retention of residual vision, (although diminished) which often improves over time ${ }^{1}$. Children with pure CVI have a normal pupillary reaction to light, and normal outcome on ophthalmological examination. CVI frequently cooccurs with other ocular disorders ${ }^{10}$, ${ }^{11}$. In fact, as many as $65 \%$ of patients with CVI may have associated ophthalmological abnormalities ${ }^{11}$.

Recently, Cioni and colleagues ${ }^{12}$ proposed that a broader range of visual impairments should be added to the general CVI category. In CVI, visual acuity is decreased because the visual pathway subserving the macula has been damaged. The macula is subserved by as much as two-thirds of the visual corte $x^{13-15}$, so loss of visual acuity is very likely to accompany any injury to this part of the brain. Some children may have associated deficits in visual field, contrast sensitivity, or ocular motility ${ }^{4}$. Other aspects of higher-order spatial vision (e.g. contour integration) are likely to be affected as well. To date, there have been few studies to systematically and quantitatively evaluate these important visual functions in patients with CVI.

Not all types of visual deficits caused by CVI will affect visual acuity. For example, in cortical visual dysfunction (CVD) ${ }^{16}$, the predominant visual deficit is not visual acuity loss, but rather disturbances in visual perception and integration. In higher-functioning children with CVI or CVD, specific visual disorders such as agnosias may be diagnosed. These include cerebral motion blindness or cerebral akinetopsia (the inability to perceive moving targets), simultanagnosia (the inability to focus on more than one visual object at a time), central achromatopsia ${ }^{16}$ (color desaturation), prosopagnosia (difficulty in recognizing faces), topographic agnosia (problems with orientation; see section on rehabilitation), and astereocognosis (difficulty with depth 
perception) ${ }^{17}$. Thus, although not all children with CVD have associated CVI, certain children with CVI (with loss of visual acuity) may show signs of $\mathrm{CVD}^{16,18}$

\section{Etiology of CVI}

The most common cause of CVI is an hypoxic-ischemic injury ${ }^{1-3,10,19,20}$. At least $60 \%$ of children with neonatal hypoxic-ischemic encephalopathy have cerebral visual impairment ${ }^{12}$. Hypoxia (lack of oxygen) or ischemia (tissue death due to loss of blood flow, and thus oxygen deprivation) in the preterm baby leads to a characteristic injury of the brain, namely periventricular leukomalacia $(\mathrm{PVL})^{21,22}$, which can be detected by MRI.

Head injury is a major cause of CVI, with half the cases of traumatic injury resulting from child abuse ${ }^{2}$. Trauma-induced $\mathrm{CVI}$ is an acute reaction to a reversible disease process, and is often accompanied by headaches, confusion, and vomiting ${ }^{23}$. Cranial injury may induce transient ischemia or edema ${ }^{23,24}$. Visual problems resulting from traumatic injury may also be chronic and lead to complete blindness ${ }^{24}$.

Other causes of CVI include: shunt failure ${ }^{10}$ (which can lead to ischemia and occipital lobe infarction), infections (bacterial meningitis ${ }^{1}$, encephalitis ${ }^{25}$, meningitis/encephalitis $^{2}$, congenital toxoplasmosis, and neonatal herpes simplex ${ }^{10}$ ), antenatal use of cocaine and amphetamines by the mother ${ }^{11}$, metabolic disease (most of the neurodegenerative diseases have the potential to disrupt cortical vision) ${ }^{26,27}$, complications of cardiac treatment (CVI has been reported after cardiac arrest and open heart surgery $)^{1,24}$, twin pregnancy $^{1,28}$, epilepsy ${ }^{29}$, and CNS developmental defects ${ }^{29}$ (lissencephaly, holoprosencephaly, and schizencephaly). CVI is virtually always associated with other serious neurological abnormalities ${ }^{2,7,11,30,31}$. Whiting and coworkers ${ }^{25}$ found evidence of abnormal cognitive development, cerebral palsy (CP), seizures, microcephaly, hydrocephalus, sensorineural hearing loss, myelomeningocoele, and progressive CNS degeneration in children with CVI.

CVI is also associated with ophthalmological abnormalities including various types of nystagmus, strabismus, and refractive error ${ }^{11,32}$ which should be corrected (e.g. with glasses) to maximize any residual vision ${ }^{2}$. Optic nerve atrophy, which itself causes vision impairment, has been seen in children with $\mathrm{CVI}^{21}$. Visual field development may be delayed in preterm children who have perinatal hypoxia-ischemia. Strabismus with a cerebral origin may also be present in these children $^{33}$.

\section{Prognosis}

Most patients with CVI will not regain normal vision ${ }^{34}$. However, improvement is usually seen over time $e^{2,3,11,34}$. Visual improvement may be rapid, particularly in cases of traumatic injury, however, usually visual recovery is gradual. Very little is known about specific prognostic findings in CVI. In general, children with CVI and extensive neurological damage have the least favorable prognosis for recovery of vision. The finding of PVL confers a particularly poor prognosis $^{35}$, compared to damage to the visual cortex only.

The prognosis for recovery of vision is probably better when the injury involves the striate cortex, not the optic radiations $^{35}$. However, involvement of basal ganglia on neuroimaging also indicates a poor developmental and visual prognosis $^{36}$. Basal ganglia disorders are almost invariably accompanied by generalized involuntary movements of the head, neck, and eyes which interfere with motor functions and vision ${ }^{21}$.

Bacterial meningitis is associated with a poorer prognosis than most other causes of $\mathrm{CVI}^{29}$. Cardiac arrest and status epilepticus are also typically associated with an unfavorable prognosis ${ }^{30}$. Fortunately, status epilepticus is now rare. Chen and colleagues ${ }^{29}$ have suggested that epilepsy is also associated with a poor prognosis. CVI is very common in infants with infantile spasms, especially when the EEG is hypsarhythmic. Abnormal visual function can result from loss of visual acuity and impaired perception.

\section{Diagnostic findings}

The clinical examination is usually sufficient to establish the diagnosis of CVI. Children with CVI who have no anterior visual pathway abnormality will have a normal eye examination but will show poor visual behavior. For example, they will fail to regard a face or to pay visual attention to their surroundings. The ophthalmoscopic examination in typical cases of CVI is normal. Some children will have both anterior visual pathway disease and CVI. In these cases, clinical judgment is used to determine whether a component of vision impairment is caused by CVI. Additional physical findings may help to clarify the diagnosis and must be taken into account in determining the overall management of the child with CVI.

Children with CVI may experience head- and eye-movement difficulties ${ }^{37}$. Abnormalities such as apraxia of eye movement and gaze palsies are common, as are abnormal pursuit eye movements. In these ocular motor disturbances, it may be difficult to distinguish the true loss of visual acuity from a disorder of eye movement that may mimic vision impairment.

Visual field defects are also common in $\mathrm{CVI}^{34}$. Measurement of visual field defects is difficult, even in normally developing children $^{38,39}$. One method used effectively to measure visual fields is the confrontational examination, which relies on the child's eye or head movement to indicate that a target has been observed.

Children with CVI exhibit slow, inefficient, and highly variable visual performance. Patients with CVI characteristically have a short visual attention $\operatorname{span}^{2,25,30,37}$. They typically see better in familiar surroundings ${ }^{4,25}$ and when they are relaxed and well rested. Other aspects of behavior associated with vision could account for this variation in visual behavior (e.g. poor motor control mimicking vision impairment or subclinical seizures interfering with visual behavior).

Color vision and perception of movement are often preserved in children with $\mathrm{CVI}^{1}$. Often, these children use peripheral vision to search for objects. They may turn their heads before reaching for an object (retinal reach) ${ }^{3,25}$ with the head turned away from the side of vision loss. Moreover, they often bring objects closer to their eyes to increase linear magnification of the object of visual interest ${ }^{2}$.

Gazing at lights is a common feature in $\mathrm{CVI}^{40}$. Paradoxically, a third of patients with CVI will exhibit photophobia, but will still gaze at lights from time to time ${ }^{41}$. Children tend to outgrow their photophobic behavior.

Investigational studies

Quantitative information about a patient's condition can be clinically useful and reassuring to patients and their families. 
A variety of techniques can be used to assess the extent of injury to the posterior visual pathways, but while a particular technique may be a good predictor of prognosis in experimental cohorts, in the case of individuals such predictions are less useful ${ }^{22,42}$.

In forced-choice preferential looking (FPL) tests, acuity is determined by noting the finest grating to which children reliably orient their gaze. Inattention or inability to gaze directly could prevent a child from following a stimulus above chance levels ${ }^{32}$. Moreover, FPL measures may be difficult to interpret in children with head- and eye-movement difficulties. Thus, failure to reliably direct gaze towards a grating card may be determined more by motor-coordination problems than by vision problems alone in the child with CVI. On the other hand, FPL testing may reveal specific defects in gaze control in conjunction with other tests.

Research on visual evoked potentials (VEPs) has focused on this method's usefulness in confirming CVI or on its prognostic value for visual outcome ${ }^{43}$. Several types of VEPs can be performed, including the transient, non-patterned flash VEP and the transient pattern reversal VEP, each of which yields information about the temporal waveform of responses to single presentations of a visual stimulus. Clarke and colleagues ${ }^{44}$ found that CVI patients with normal flash VEPs had a good prognosis for improvement. However, flash VEP may not accurately assess higher levels of visual processing. Pattern VEPs are more useful for monitoring visual development and rehabilitation in children ${ }^{45}$. Steady state VEPs show promising potential for quantification of visual loss in CVI and offer the advantage of testing several types of visual function (e.g. contrast sensitivity, grating acuity, vernier acuity) ${ }^{46}$. The VEP provides general information about geniculocalcarine dysfunction and occipital responses to photic stimuli $^{1}$; the EEG can be interpreted in association with VEPs. The presence of normal alpha rhythm, superimposed on a normal background, rules out cortical visual impairment and homonymous hemianopia due to cortical lesions ${ }^{31}$.

Clinical assessment can be supported with brain imaging studies. Neuroimaging of the brain can be used to confirm the clinical diagnosis of $\mathrm{CVI}^{4}$. MRI is often used to detect PVL in the first days of life, although the child's visual outcome cannot be accurately predicted based on neuroimaging findings ${ }^{33}$. Ultrasound, which is portable and non-invasive, is also used to detect PVL in the first days of life and may be more sensitive than MRI during this period. Eken and coworkers ${ }^{47}$ found that ultrasound could be used to correlate structural abnormalities with poor visual outcome.

MRI is also used to assess asphyxia in neonates ${ }^{48}$ and may be the best predictor of outcome in the first week following injury. A normal MRI correlates with normal vision, although an abnormal MRI finding does not necessarily indicate loss of visual acuity ${ }^{49}$. Finally, MRI may show selective damage to periventricular white matter, with a less favorable prognosis for visual recovery ${ }^{35,49}$.

SPECT and PET scans have been used to investigate changes in cerebral blood flow ${ }^{50}$. These tools may be better at predicting outcome than MRI but have not been widely used, mainly because PET requires delivery of a small amount of a radioactive isotope. Functional magnetic resonance imaging (fMRI) shows promise as a diagnostic tool. The fMRI measure demonstrates areas of the brain that are metabolically active. However, fMRI requires an alert, immobile, cooperative patient and, therefore, has limited use in children.

\section{Clinical management}

The identification of other neurological disorders associated with CVI is an important component of management. Many children with CVI have motor complications, including disturbances of ocular motor function. These motor control problems complicate the interpretation of behavioral signs of vision in children with CVI, and pose potential problems in the diagnosis and management of CVI.

Prompt management of any medical problem that could interfere with hearing (e.g. otitis media) is essential, since children with CVI may rely extensively on their other sensory functions. Patients with CVI frequently have complex epileptic disorders. Anticonvulsant drugs must be carefully selected, with continuous monitoring of the blood levels, in order to avoid excessive sedation.

Sleep disorders in visually impaired children have complex etiologies ${ }^{51}$ and have been associated with painful esophageal reflux and orthopedic problems. Chronic sleep disturbances in children with CVI who have multiple disabilities may be treated with hypnotics or melatonin ${ }^{52}$.

Poor feeding skills and recurrent problems with aspiration are common because of difficulties encountered with foods that require chewing (partly a visually learned skill) and swallowing. Affected children frequently have CP with its associated orthopedic problems, such as subluxated or dislocated hips. These children will benefit from a comfortable and functional seating arrangement, which may help to promote cooperation during visual rehabilitation.

Many patients with severe developmental delays are readily overstimulated in an uncontrolled environment. The inability to cope efficiently in this environment may provoke sleep disruptions, which may ultimately trigger self-abusive behavior. The neurological disorder of physical self-abuse is often overlooked and can only be treated effectively by modifying home and school environments.

Children with neurodevelopmental disabilities and their families require the services of a multidisciplinary team specialized in the development of children with visual impairments. These specialists can provide an accurate evaluation of the patient's condition and formulate an individual program plan to monitor the child's progress ${ }^{53}$. Major medical centers now have experienced specialists who deal with the needs of children with disabilities. The effectiveness of the team approach may ultimately provide a better developmental outcome for the child ${ }^{54}$.

\section{Rehabilitation program}

The goal of visual rehabilitation is to maximize the use of functional residual vision. Early assessment is critical ${ }^{2}$. The misdiagnosis of CVI, either as a behavioral difficulty or a learning disability, can delay remediation and result in significant educational delays that leave emotional sequelae ${ }^{55}$.

Visual and cognitive development are closely related ${ }^{54}$. Younger patients, who have not reached developmental maturity will require special learning environments that promote maximum stimulation of residual vision. Such environmental stimulation may help to prevent developmental delays ${ }^{56}$. Research indicates that developmental milestones that normally require vision ('reaching' and 'walking') ${ }^{57}$ are often delayed in children with visual impairment, even in the 
absence of other disabilities.

Although traditional educational settings strive to provide stimulation and diversity to encourage children's developmental growth, a simplified visual environment is more beneficial to children with CVI because it forces them to focus attention on a particular visual stimulus ${ }^{1,2}$. In these children, color, high contrast, and the use of motion may facilitate visual recognition of an object ${ }^{1,3,37}$. Reinforcement with tactile and verbal stimulation is also important. The 'ritualization' of tasks performed in standardized fashion at the same time every day may also be helpful ${ }^{2}$. Consistency of the environment may be particularly helpful for those children with orientation problems, known as topographical agnosia ${ }^{58}$ which is characterized by a poor sense of location or direction. This condition may affect children who are ambulatory or have mobility in a wheelchair.

Children with CVI appear to be at a disadvantage when performing reading and mathematical tasks, although this may only be partly attributed to intellectual capacity and visual impairment ${ }^{4}$. Reading difficulties may derive in part from the 'crowding' effect, which is the child's inability to see a symbol when it is flanked by other symbols that crowd the visual field ${ }^{2,37}$. Sliding a finger along a line of text may facilitate reading in children with CVI who have developed higher-functioning skills. Pointing at distant targets may improve tracking ${ }^{16}$.

Some studies suggest that children with visual impairments may learn to express themselves before sighted children ${ }^{4}$, although other studies have reported that language development is initially delayed ${ }^{59}$. Children with CVI are usually delayed in language skills. Learning to write legibly can also be difficult for children with visual impairment ${ }^{60}$

Intelligence tests in children with visual impairment may not reveal their true potential if their scores are compared to those of either sighted or blind children ${ }^{61}$. Therefore, assessment of children with visual impairment should be performed by experienced specialists knowledgeable about the development of children with visual impairments. There is a trend in the USA to integrate children with disabilities into the mainstream classroom. However, children with CVI will benefit more from the implementation of rehabilitation programs that provide a streamlined, simplified, visual environment ${ }^{2}$.

\section{Conclusion}

Central to the diagnosis of CVI is loss of visual acuity. Future research in CVI will continue to elucidate the various patterns of vision loss that accompany loss of visual acuity. To this effect, better diagnostic tools are needed to accurately define deficits in pattern vision and vision processing (higher-order vision). Currently, there is no precise treatment for CVI, and many rehabilitative measures are unproven. Clearly, there is a great need for additional research on treatment and management of this common and complex disorder.

\section{Accepted for publication 15th June 2000.}

\section{Acknowledgements}

Dr Good was supported by National Eye Institute grant EY00384.

\section{References}

1. Baker-Nobles L, Rutherford A. (1995) Understanding cortical visual impairment in children. American Journal of Occupational Therapy 49: 899-903.
2. Groenveld M, Jan JE, Leader P. (1990) Observations on the habilitation of children with cortical visual impairment. Journal of Visual Impairment and Blindness 84: 11-5.

3. Good WV, Jan JE, deSa L, Barkovich AJ, Groenveld M, Hoyt CS (1994) Cortical visual impairment in children: a major review. Survey of Opbthalmology 38: 351-64.

4. Jacobson L, Ek U, Fernell E, Flodmark O, Broberger U. (1996) Visual impairment in preterm children with periventricular leukomalacia: visual, cognitive and neuropaediatric characteristics related to cerebral imaging. Developmental Medicine \& Child Neurology 38: 724-35.

5. Rosenberg T, Flage T, Hansen E, Riise R, Rudanko SL, Vigosson $\mathrm{G}$, Tornqvist K. (1996) Incidence of registered visual impairment in the Nordic child population. British Journal of Ophthalmology 80: 49-53.

6. Gilbert CE, Canovas R, Kocksch de Canovas R, Foster A. (1994) Causes of blindness and severe visual impairment in children in Chile. Developmental Medicine \& Child Neurology 36: 326-33.

7. Rogers M. (1996) Visual impairment in Liverpool: prevalence and morbidity. Archives of the Disabled Child 94: 299-303.

8. National Perinatal Epidemiology Unit. (1988) Oxford Register of Early Childbood Impairments Annual Report. Oxford: Radcliffe Infirmary. p 32-6.

9. Murphy D, Good WV. (1997) The epidemiology of blindness in children. Paper presented at the American Academy of Opbthalmology. 157. (Abstract).

10. Flodmark O, Jan JE, Wong P. (1990) Computed tomography of the brains of children with cortical visual impairment. Developmental Medicine \& Child Neurology 32: 611-20.

11. Huo R, Burden S, Hoyt CS, Good WV. (1999) Chronic cortical visual impairment in children: aetiology, prognosis, and associated neurological deficits. British Journal of Opthamology 83:670-5.

12. Cioni G, Fazzi B, Ipata AE, Canapicchi R, van Hof-van Duin J. (1996) Correlation between cerebral visual impairment and magnetic resonance imaging in children with neonatal encephalopathy. Developmental Medicine $\&$ Cbild Neurology 38: 120-32.

13. Holmes G, Lister WT. (1916) Disturbances of vision from cerebral lesions with special reference to the cortical representation of the macula. Brain 39: 34-37.

14. Horton JC, Hoyt WF. (1991) The representation of the visual field in human striate cortex. Archives of Opbthalmology 109: 816-24.

15. Wong AMF, Sharpe JA. (1999) Representation of the visual field in the human occipital cortex: a magnetic imaging and perimetric correlation. Archives of Opbthalmology 11\%: 208-17.

16. Dutton GN. (1994) Cognitive visual dysfunction. British Journal of Opbthalmology \%8: 723-26.

17. Ahmed M, Dutton GN. (1996) Cognitive visual dysfunction in a child with cerebral damage. Developmental Medicine $\mathcal{E}$ Child Neurology 38: 736-43.

18. Houliston MJ, Taguri AH, Dutton GN, Hajivassiciou C, Young DE. (1999) Evidence of cognitive visual problems in children with hydrocephalus: a structured clinical history-taking strategy. Developmental Medicine \& Child Neurology 41: 298-306.

19. Goggin M, O'Keefe M. (1991) Childhood blindness in the Republic of Ireland: a national survey. British Journal of Ophthalmology \%5: 425-9.

20. Taylor MJ, McCulloch DL. (1991) Prognostic value of VEPs in young children with acute onset of cortical blindness. Pediatric Neurology \%: 111-5.

21. Roland EH, Jan JE, Hill A, Wong PK. (1986) Cortical visual impairment following birth asphyxia. Pediatric Neurology 12: $133-7$.

22. Pike MG, Holmstrom G, de Vries LS, Pennock JM, Drew KJ, Sonksen PM, Dubowitz LM. (1994) Patterns of visual impairment associated with lesions of the preterm infant brain. Developmental Medicine \& Child Neurology 36: 849-62.

23. Sakas DE, Whittaker KW, Whitwell HL, Singounas EG. (1997) Syndromes of posttraumatic neurological deterioration in children with no focal lesions revealed by cerebral imaging: evidence for a trigeminovascular pathophysiology. Neurosurgery 41: 661-7. 
24. Rodriguez A, Lozano JA, del Pozo D, Homar Paez J. (1993) Post-traumatic transient cortical blindness. International Ophthalmology 1\%: 277-83.

25. Whiting S, Jan JE, Wong PK, Flodmark O, Farrell K, McCormick AQ. (1985) Permanent cortical visual impairment in children. Developmental Medicine \& Child Neurology 2\%: 730-9.

26. Schaumberg HH, Powers JM, Raine CS, Suzuki K, Richardson JO. (1975) Adrenoleucodystrophy: a clinical and pathological study of 17 cases. Archives of Neurology 32: 577-91.

27. Wray SH, Cogan DG, Kuwabara T, Schaumberg HH, Powers JM. (1976) Adrenoleucodystrophy with disease of the eye and optic nerve. American Journal of Opbthalmology 82: 480-5.

28. Good WV, Brodsky MC, Angtuaco TL, Ferriero DM, Stephens DC 3rd, Khakoo Y. (1996) Cortical visual impairment caused by twin pregnancy. American Journal of Opbthalmology 122: 709-16.

29. Chen TC, Weinberg MH, Catalano RA, Simon JW, Wagle WA (1992) Development of object vision in infants with permanent cortical visual impairment. American Journal of Ophthalmology 114: 575-8.

30. Wong VCN. (1991) Cortical blindness in children: a study of etiology and prognosis. Pediatric Neurology \%: 178-85.

31. Jan JE, Wong PK. (1988) Behaviour of the alpha rhythm in electroencephalograms of visually impaired children. Developmental Medicine \& Child Neurology 30: 444-50.

32. Birch EE, Bane MC. (1991) Forced-choice preferential looking acuity of children with cortical visual impairment. Developmental Medicine \& Child Neurology 33: 722-9.

33. Cioni G, Fazzi B, Coluccini M, Bartalena L, Boldrini A, van Hof-van Duin J. (1997) Cerebral visual impairment in preterm infants with periventricular leukomalacia. Pediatric Neurology 1\%: 331-8.

34. Groenendaal F, van Hof-van Duin J. (1992) Visual deficits and improvements in children after perinatal hypoxia. Journal of Visual Impairment and Blindness 86: 215-8.

35. Lambert SR, Hoyt CS, Jan JE, Barkovich J, Flodmark O. (1987) Visual recovery from hypoxic cortical blindness during childhood. Computed tomographic and magnetic resonance imaging predictors. Archives of Opbthalmology 105: 1371-7.

36. Mercuri E, Atkinson J, Braddick O, Anker S, Cowan F, Rutherford M, Pennock J, Dubowitz L. (1997) Visual function in full-term infants with hypoxic-ischaemic encephalopathy. Neuropediatrics 28: 155-61.

37. Jan JE, Groenveld M. (1993) Visual behaviors and adaptations associated with cortical and ocular impairment in children. Journal of Visual Impairment and Blindness: 101-5.

38. Harvey EM, Dobson V, Narter DB. (1997) The influence of a central stimulus on visual field measurements in children from 3.5 to 30 months of age. Optometry and Visual Science 74: $768-74$

39. Dobson V, Brown AM, Harvey EM, Narter DB. (1998) Visual field extent in children 3.5-30 months of age tested with a double-arc LED perimeter. Vision Research 38: 2743-60.

40. Jan JE, Groenveld M, Sykanda AM. (1990) Light-gazing by visually impaired children. Developmental Medicine $\&$ Child Neurology 32: 755-9.

41. Jan JE, Groenveld M, Anderson DP. (1993) Photophobia and cortical visual impairment. Developmental Medicine $\&$ Child Neurology 35: 473-7.

42. Van Hof-van Duin J, Cioni G, Bertuccelli B, Fazzi B, Romano C, Boldrini A. (1998) Visual outcome at 5 years of newborn infants at risk of cerebral visual impairment. Developmental Medicine E Child Neurology 40: 302-9.

43. Granet DB, Hertle RW, Quinn GE, Breton ME. (1993) The visual-evoked response in infants with central visual impairment. American Journal of Opbthalmology 116: 437-43.

44. Clarke MP, Mitchell KW, Gibson M. (1997) The prognostic value of flash visual evoked potentials in the assessment of non-ocular visual impairment in infancy. Eye 11: 398-402.

45. Taylor MJ, McCulloch DL. (1991) Prognostic value of VEPs in young children with acute onset of cortical blindness Pediatric Neurology \%: 111-5.

46. Skoczenski A, Norcia AM. (1999) Development of VEP vernier acuity and grating acuity in human infants. Investigative Opbthalmology and Visual Science 40: 2411-7.
47. Eken P, van Nieuwenhuizen O, van der Graaf Y, Schalij-Delfos NE, de Vries LS. (1994) Relation between neonatal cranial ultrasound abnormalities and cerebral visual impairment in infancy. Developmental Medicine E Child Neurology 36: 3-15.

48. Barkovich AJ, Hajnal BL, Vigneron D, Sola A, Partridge JC, Allen F, Ferriero DM. (1998) Prediction of neuromotor outcome in perinatal asphyxia: Evaluation of MR scoring systems. American Journal of Neuroradiology 19: 143-9.

49. Casteels I, Demaerel P, Spileers W, Lagae L, Missotten L, Casaer P. (1997) Cortical visual impairment following perinatal hypoxia: clinicoradiologic correlation using magnetic resonance imaging. Journal of Pediatric Ophthalmology and Strabismus 34: 297-305.

50. Silverman IE, Galetta SL, Gray LG, Moster M, Atlas SW, Maurer AH, Alavi A. (1993) SPECT in patients with cortical visual loss. Journal of Nuclear Medicine 34: 1447-51.

51. Stores G, Ramchandani P. (1999) Sleep disorders in visually impaired children. Developmental Medicine $\&$ Child Neurology 41: 348-52.

52. Jan JE, Freeman RD, Fast DK. (1999) Melatonin treatment of sleep-wake cycle disorders in children and adolescents. Developmental Medicine $\&$ Child Neurology 41: 491-501.

53. Hyvärinen L. (1995) Considerations in evaluation and treatment of the child with low vision. American Journal of Occupational Therapy 49: 891-7.

54. Freeman RD, Goetz E, Richards DP, Groenveld M. (1991) Definers of negative predictions: results of a 14-year follow-up study. Journal of Visual Impairment and Blindness 85: 365-70.

55. Sonksen PM. (1993) The assessment of vision in the preschool child. Archives of the Disabled Child 68: 513-6.

56. Cass HD, Sonksen PM, McConachie HR. (1994) Developmental setback in severe visual impairment. Archives of the Disabled Child \%0: 192-96.

57. Moller MA. (1993) Working with visually impaired children and their families. Pediatric Clinics of Nortb America 40: 881-90.

58. Dutton G, Ballantyne J, Boyd G, Bradnam M, Day R, McCulloch D, Mackie R, Phillips S, Saunders K. (1996) Cortical visual dysfunction in children: a clinical study. Eye 10: 302-9

59. McConachie HR, Moore V. (1994) Early expressive language of severely visually impaired children. Developmental Medicine E Child Neurology 36: 230-40.

60. Moore V, Law J. (1990) Copying ability of preschool children with delayed language development. Developmental Medicine E Child Neurology 32: 249-57.

61. Dekker R. (1993) Visually impaired children and haptic intelligence test scores: intelligence tests for visually impaired children (ITVIC) Developmental Medicine \& Child Neurology 35: 478-89. 